\title{
In situ effective snow grain size mapping using a compact hyperspectral imager
}

\section{Article}

Cite this article: Donahue C, Skiles SM, Hammonds K (2021). In situ effective snow grain size mapping using a compact hyperspectral imager. Journal of Glaciology 67 (261), 49-57. https://doi.org/10.1017/ jog.2020.68

Received: 17 February 2020

Revised: 21 July 2020

Accepted: 22 July 2020

First published online: 18 August 2020

Keywords:

Crystal growth; remote sensing; snow; snow metamorphosis

\section{Author for correspondence:}

Christopher Donahue,

E-mail: christopher.donahue2@student. montana.edu

\section{Christopher Donahue $^{1}\left(\mathbb{D}\right.$, S. McKenzie Skiles ${ }^{2}$ (D) and Kevin Hammonds ${ }^{1}$ (D)}

${ }^{1}$ Department of Civil Engineering, Montana State University, Bozeman, MT, USA and ${ }^{2}$ Department of Geography, University of Utah, Salt Lake City, UT, USA

\section{Abstract}

Effective snow grain radius $\left(r_{\mathrm{e}}\right)$ is mapped at high resolution using near-infrared hyperspectral imaging (NIR-HSI). The NIR-HSI method can be used to quantify $r_{\mathrm{e}}$ spatial variability, change in $r_{\mathrm{e}}$ due to metamorphism, and visualize water percolation in the snowpack. Results are presented for three different laboratory-prepared snow samples (homogeneous, ice lens, fine grains over coarse grains), the sidewalls of which were imaged before and after melt induced by a solar lamp. The spectral reflectance in each $\sim 3 \mathrm{~mm}$ pixel was inverted for $r_{\mathrm{e}}$ using the scaled band area of the ice absorption feature centered at $1030 \mathrm{~nm}$, producing $r_{\mathrm{e}}$ maps consisting of 54740 pixels. All snow samples exhibited grain coarsening post-melt as the result of wet snow metamorphism, which is quantified by the change in $r_{\mathrm{e}}$ distributions from pre- and post-melt images. The NIRHSI method was compared to $r_{\mathrm{e}}$ retrievals from a field spectrometer and X-ray computed microtomography (micro-CT), resulting in the spectrometer having the same mean $r_{\mathrm{e}}$ and micro-CT having $23.9 \%$ higher mean $r_{\mathrm{e}}$ than the hyperspectral imager. As compact hyperspectral imagers become more widely available, this method may be a valuable tool for assessing $r_{\mathrm{e}}$ spatial variability and snow metamorphism in field and laboratory settings.

\section{Introduction}

Snow grain size is a physical property of snow that exerts controls on snow reflectance (Wiscombe and Warren, 1980), and is used to characterize mechanisms of snow metamorphism and stratigraphy (Colbeck, 1991). At the surface, characterizing the snow grain size is critical for modeling snow albedo, a primary control for snow energy balance and snowmelt timing (Marks and Dozier, 1992). Whereas stratigraphy, or the vertical distribution of snow grain size, is critical for modeling microwave radiative transfer (Brucker and others, 2011) and understanding mechanical (Pielmeier and Schneebeli, 2003) and thermodynamic (Hammonds and others, 2015) properties of a snowpack.

There are multiple ways to characterize snow grain size. The classical snow grain size, defined as the largest extension $(\mathrm{mm})$ of a snow grain, is most simply observed using a crystal card and hand lens (Fierz and others, 2009). Whereas the specific surface area (SSA) is defined as the surface area per unit volume and is measured using stereology methods. Snow grain size can also be described as an effective grain radius $\left(r_{\mathrm{e}}\right)$, which is used to approximate the scattering and absorption properties of snow. Grenfell and Warren (1999) demonstrated that a collection of spherical ice particles with the same volume-to-surface area ratio as snow (i.e. characterized with stereology methods) could be used to model hemispherical reflectance. The effective grain radius is directly related to SSA by:

$$
\mathrm{SSA}=\frac{3}{r_{\mathrm{e}}} .
$$

In the near-infrared (NIR) wavelengths $(800-2500 \mathrm{~nm})$, snow spectral reflectance has a characteristic decline due to the increasing absorptivity of ice at these wavelengths. Reflectance, therefore, has an inverse relationship with grain size in this wavelength range because absorption is a function of the amount of ice per unit volume. For the same snow layer, NIR reflectance typically declines over time because grain coarsening occurs due to snow metamorphism. Leveraging this relationship, methods have been developed to retrieve $r_{\mathrm{e}}$ from various instruments that measure snow reflectance by relating the magnitude of NIR reflectance, or area/ depth of distinct ice absorption features, to $r_{\mathrm{e}}$. Broadly, these can be classified as either discrete measurements or mapping techniques, and further characterized by the spectral range and resolution of the instrument.

At the landscape scale, remote-sensing techniques are used to map $r_{\mathrm{e}}$ on a pixel-wise basis from surface reflectance. It has been shown that spaceborne multispectral sensors, like the Moderate Resolution Imaging Spectroradiometer (MODIS), can be used to map $r_{\mathrm{e}}$ using band ratios or spectral unmixing (Painter and others, 2009, 2012). These satellite datasets have value due to their record length, spatial coverage and temporal resolution, but high degrees of uncertainty result from mixed pixels and low spectral resolutions. For airborne imaging spectrometers, like the Airborne Visible/Infrared Imaging Spectrometer (AVIRIS), $r_{\mathrm{e}}$ is mapped at the scale of tens of meters by inverting the shape of ice absorption features resolved in spectral reflectance with radiative transfer modelling (Nolin and Dozier, 2000; Seidel and others, 2016). Spatial and spectral resolution are much higher, and uncertainty 
lower compared to spaceborne platforms, but due to logistics and cost, airborne datasets are very limited in spatial extent and temporal coverage.

At the local scale (i.e. laboratory, plot, snow pit) field spectrometers are used to retrieve discrete measurements of $r_{\mathrm{e}}$ from surface spectral reflectance, or along the vertical snow profile $(\sim 2 \mathrm{~cm})$ when coupled to a contact probe, known as contact spectroscopy (Painter and others, 2007). Lower spectral resolution field instruments have also been developed to measure $r_{\mathrm{e}}$ from small discrete snow samples $(\sim 3 \mathrm{~cm})$ using an integrating sphere and NIR light source, from which reflectance is empirically related, or calibrated, to known reflectance from a range of snow grain sizes. Examples of these instruments include DUFISSS (Gallet and others, 2009), IceCube (Zuanon and A2 Photonic Sensors, 2013) and Infrasnow (Gergely and others, 2014). Similarly, a vertical profiler, POSSSUM, has been developed to measure SSA profiles in one-dimension from measurements of reflectance at $1310 \mathrm{~nm}$ with a vertical resolution of $10 \mathrm{~mm}$ (Arnaud and others, 2011). Discrete measurements can be time consuming to collect, are limited to instrument field of view/sample size, and cannot efficiently capture spatial variability. This can be addressed by mapping $r_{\mathrm{e}}$, which has been demonstrated using a digital NIR photographic method (Matzl and Schneebeli, 2006). NIR photography correlates snow reflectance from 840 to $940 \mathrm{~nm}$ to SSA, measured using stereology methods, and is high resolution $(\sim 1 \mathrm{~mm})$. However, because this method uses an empirical calibration from reflectance across a single short NIR wavelength range, it is sensitive to illumination conditions and is not easily transferable between camera sensors. Currently, there is no high spatial and high spectral resolution method for mapping $r_{\mathrm{e}}$ at the local scale.

This gap is addressed here with NIR hyperspectral imaging (NIR-HSI) using a Resonon Pika NIR-320 compact hyperspectral imager and rotational stage mounted on a tripod, shown in Figure 1, to map the NIR spectral reflectance of snow. From the per pixel spectral reflectance, $r_{\mathrm{e}}$ is retrieved by applying the Nolin-Dozier (N-D) scaled band area inversion technique (Nolin and Dozier, 2000). To demonstrate this method, we present results from three laboratory-prepared snow samples with varying initial conditions that underwent induced melt and subsequent wet snow metamorphism. In general, when liquid water is introduced into a snowpack, snow grains begin rapid metamorphism and larger snow grains tend to grow at the expense of smaller grains (Marsh, 1987). By mapping $r_{\mathrm{e}}$ before and after melt, we demonstrate the ability of this technique to map snow stratigraphy for a wide range of $r_{\mathrm{e}}$ values. Further, it is shown that mapping $r_{\mathrm{e}}$ can be used to study wet snow metamorphism processes, filling a gap in current methodologies.

In previous laboratory experiments investigating liquid water flow through snow and wet snow metamorphism, dye tracers have been used to visualize water transport (Gerdel, 1954), preferential flow channels (Schneebeli, 1995) and capillary barriers (Avanzi and others, 2016). With dye tracers, the flow and pooling of water can be visualized, but quantification of grain growth with a hand lens or stereology methods is difficult and time consuming. In other laboratory work, X-ray computed microtomography (micro-CT) has been used to investigate preferential flow (Avanzi and others, 2017). Although snow grain size and many other snow properties can be accurately measured with micro-CT, the spatial resolution is often limited by the sample size limitations of the instrument.

In the results shown here, we demonstrate that when using the NIR-HSI method to map $r_{\mathrm{e}}$, preferential flow can be visualized, water pooling at interfaces can be identified, and grain growth at the laboratory or snow pit scale can be more effectively quantified, all at a higher spatial resolution than these previous methods. Additionally, a series of experiments were conducted to assess the sensitivity of the NIR-HSI method to illumination angle and general repeatability using homogeneous unperturbed snow samples. In all experiments, our results are validated by comparing the $r_{\mathrm{e}}$ retrieval from the hyperspectral imager to established in situ field spectrometer (Painter and others, 2007) and micro-CT (Flin and others, 2004; Schneebeli and Sokratov, 2004) retrieval methods for quantifying $r_{\mathrm{e}}$.

\section{Methods}

\subsection{Instrument}

For the NIR-HSI method presented here, a Resonon Inc. (Bozeman, MT, USA) Pika NIR-320 near-infrared hyperspectral imager was used. This imager is a compact line-scan imager (also called a 'push-broom' scanner), meaning 2-D images are constructed by collecting the image line by line while the imager translates or rotates relative to the sample (https://resonon.com/). This instrument covers the spectral range from 900 to $1700 \mathrm{~nm}$ across 164 channels, resulting in a spectral resolution of $4.9 \mathrm{~nm}$, at 14-bit radiometric resolution. The imager has 320 spatial channels with a sensor pixel size of $30 \mu \mathrm{m}$. The operating temperature of the imager, per its datasheet, is $5-40^{\circ} \mathrm{C}$. Following the experiments at $-5^{\circ} \mathrm{C}$, it was confirmed by the manufacturer that the optical alignment had not deteriorated, indicating the initial calibration was not compromised by cold temperatures.

The scanner was mounted onto the Resonon rotational stage for imaging perpendicular to the snow sample sidewall at a distance of $100 \mathrm{~cm}$ (Fig. 1), resulting in a spatial resolution of $\sim 3 \mathrm{~mm}$. Two 500-watt halogen lamps mounted on a tripod were located perpendicular to the snow sample for equal illumination of the sidewall. For calibration from digital number to the bidirectional reflectance factor, a Spectralon $99 \%$ diffuse white reference reflectance panel was placed in the scene of each hyperspectral image. Using the proprietary Resonon Spectronon software, snow sample sidewalls were imaged with an integration time of $8.05 \mathrm{~ms}$, frame rate of $124 \mathrm{~Hz}$ and a scanning speed of $7.7 \mathrm{deg} \mathrm{s}^{-1}$. Before processing, each image was cropped such that only the exposed face of the snow sample was used for the retrieval of $r_{\mathrm{e}}$.

\subsection{Effective grain radius retrieval}

Following N-D, the spectra at each pixel in the hyperspectral image was inverted for $r_{\mathrm{e}}$ using the scaled band area $\left(A_{\mathrm{b}}\right)$, which is calculated by integrating the continuum-scaled ice absorption feature centered at $\lambda=1030 \mathrm{~nm}$ (Nolin and Dozier, 2000).

$$
A_{\mathrm{b}}=\int_{\lambda=984 \mathrm{~nm}}^{\lambda=1087 \mathrm{~nm}} \frac{R_{\text {cont }, \lambda}-R_{\text {snow }, \lambda}}{R_{\text {cont }, \lambda}} \mathrm{d} \lambda,
$$

where $R_{\text {snow }, \lambda}$ is the measured snow reflectance at wavelength $\lambda$ and $R_{\text {cont }, \lambda}$ is the continuum reflectance at wavelength $\lambda$, defined as the slope between the shoulders of the ice absorption feature, which represents what the reflectance spectrum would be in the absence of ice absorption. To help visualize the scaled band area and continuum reflectance, Figure 2a illustrates them as the shaded gray area and solid red line, respectively. Here, the end points are the reflectance values from the Pika NIR-320 bands centered at $\lambda=$ $984 \mathrm{~nm}$ and $\lambda=1087 \mathrm{~nm}$, with the integral taken over the 21 bands between end points. We chose to use $984 \mathrm{~nm}$, which narrows the range relative to $\mathrm{N}-\mathrm{D}$, because it is not impacted by noise at the lower limit of the Pika NIR-320 hyperspectral sensor (900 nm). By integrating relative to the continuum reflectance, the grain size 


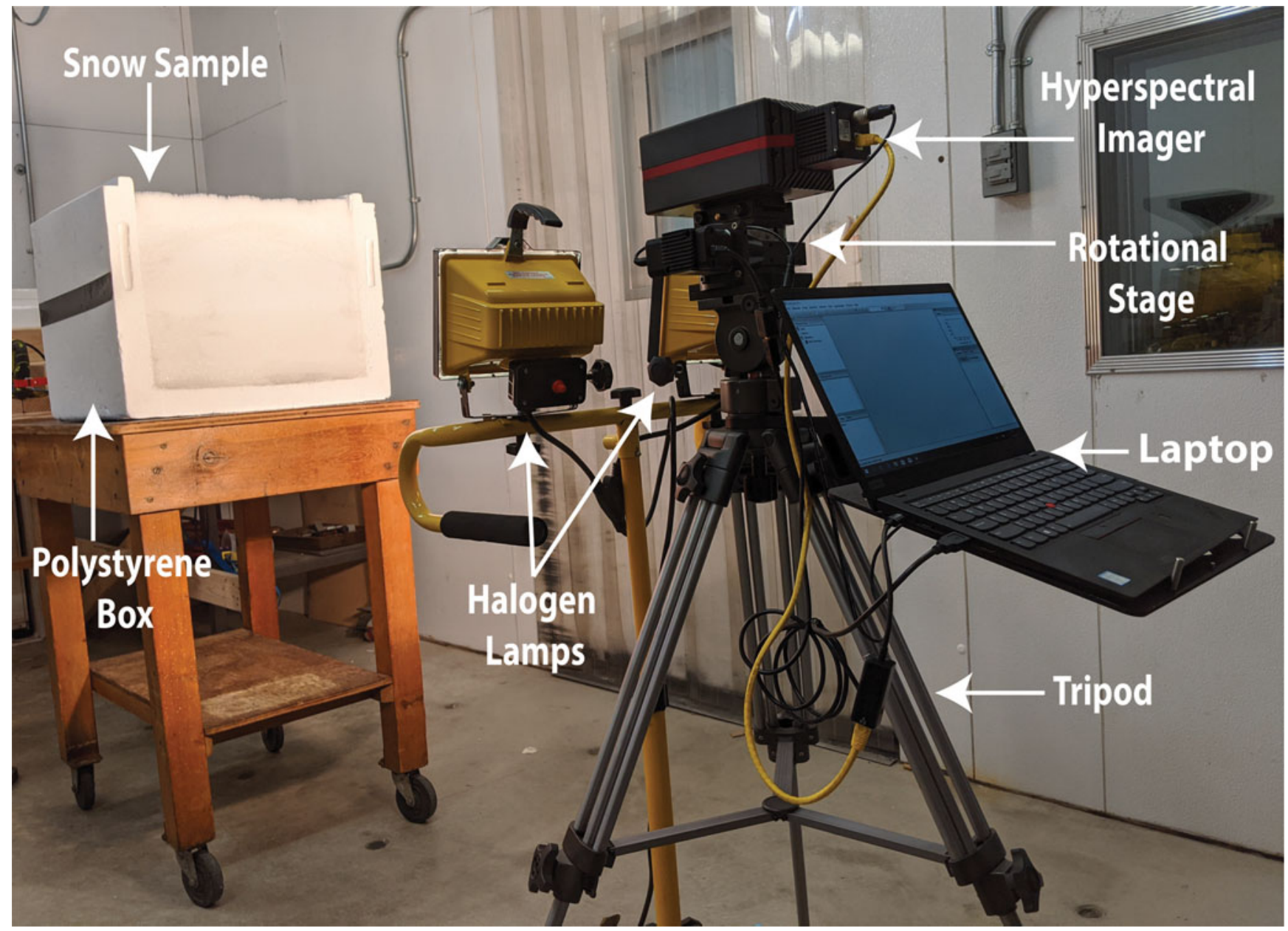

Fig. 1. Laboratory setup showing a laboratory-prepared snow sample, two halogen lamps, a Resonon Pika NIR-320 hyperspectral imager and a rotational stage mounted on a tripod inside a cold room. The hyperspectral imager is located $100 \mathrm{~cm}$ from the exposed snow sample sidewall.
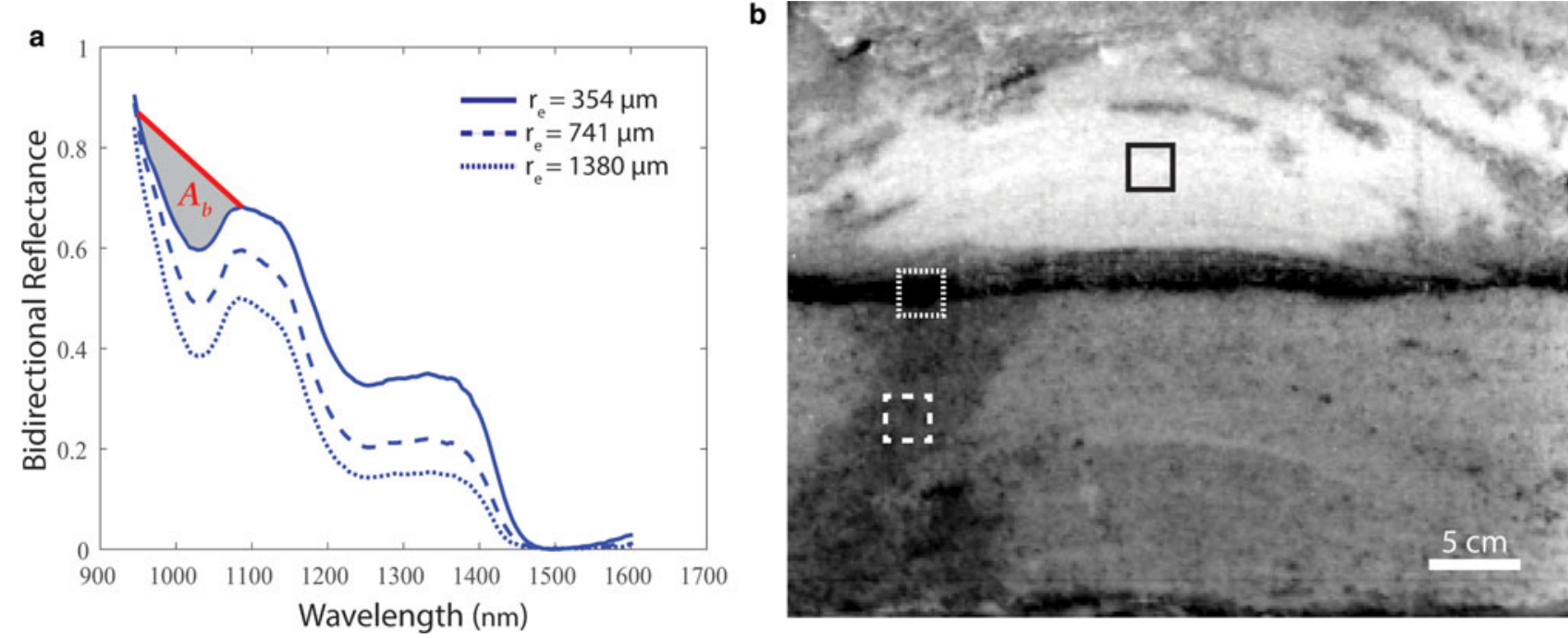

Fig. 2. (a) Three snow spectra are plotted from a single pixel located within the boxes of (b). For the solid line spectrum, an example of the scaled band area $\left(A_{b}\right)$ is shaded gray, which corresponds to an effective grain radius $\left(r_{\mathrm{e}}\right)$ of $354 \mu \mathrm{m}$. The continuum reflectance, shown as the red line, is the slope between the shoulders of the ice absorption feature $(\lambda=984 \mathrm{~nm}$ and $\lambda=1087 \mathrm{~nm})$. (b) Gray scale near-infrared image of the post-melt fine grain over coarse grains snow sample at $\lambda=1030$ $\mathrm{nm}$ taken with the Resonon Pika NIR-320 hyperspectral imager. Each box represents an area with a different $r_{\mathrm{e}}$.

estimates are independent of the magnitude of reflectance (Nolin and Dozier, 2000). This means that $r_{\mathrm{e}}$ retrievals should also be relatively insensitive to variability in illumination that can result from slight deviations of the imager and light source from normal incidence, relative to the snow sample sidewall and Spectralon panel, although this was assessed separately (see Section 2.4.2).

Nolin and Dozier (2000) validated their model with a crystal card, hand lens and stereology measurements, finding that 
retrieved $r_{\mathrm{e}}$ matched measurements with a correlation coefficient of $r=0.997$. They also assessed the sensitivity of the method to instrument noise, finding that retrieved $r_{\mathrm{e}}$ between 30 and 900 $\mu \mathrm{m}$ could vary by $\pm 10-50 \mu \mathrm{m}$. The signal-to-noise ratio (SNR) used for AVIRIS in the study was 100, whereas the SNR for the Resonon Pika NIR-320 is 1885 . With the better SNR and higher spectral resolution of the Pika NIR-320, the NIR-HSI method should be more robust with respect to sensor noise, when compared to the N-D method applied to AVIRIS.

In place of the Discrete Ordinates Radiative Transfer Program, or DISORT (Stamnes and others, 1988) used by N-D, the theoretical snow spectra in this study were modeled with the Snow, Ice, and Aerosol Radiative Transfer Model, or SNICAR (Flanner and others, 2007). SNICAR simulates radiative transfer through a snowpack comprised of spherical grains of ice at a given grain size and illumination geometry using the updated ice optical property compilation of Warren and Brandt (2008). Model inputs include illumination angle, $r_{\mathrm{e}}(\mu \mathrm{m})$, snow layer thickness $(\mathrm{m})$, snow density $\left(\mathrm{kg} \mathrm{m}^{-3}\right)$ and when appropriate, concentrations of light-absorbing particles (ppb). In this case, we simulated snow directional-hemispherical reflectance for clean snow only, by running the model iteratively through $r_{\mathrm{e}}$ values between 30 and $1500 \mu \mathrm{m}$. The other forcing inputs were held constant at values that best represent the bulk characteristics of the snow sample; $300 \mathrm{~kg} \mathrm{~m}^{-3}$ snow density, $0^{\circ}$ illumination angle (light source perpendicular to snow surface) and a single optically thick snow layer. We did not vary the snow layer thickness because light penetration in the NIR is very shallow $(0.5-3 \mathrm{~cm})$ and grain size can be considered mostly homogeneous at this scale (Warren, 1982; Nolin and Dozier, 2000). We also did not vary snow density because density has little to no effect on snow reflectance (Bohren and Beschta, 1979).

To help visualize the NIR hyperspectral image, a grayscale image at $\lambda=1030 \mathrm{~nm}$ is shown in Figure $2 \mathrm{~b}$. In each pixel, $r_{\mathrm{e}}$ is determined through the best match to the theoretical scaled band area values contained within a lookup table (Supplementary Material Table S1), populated from modeled snow spectra using SNICAR. In Figure 2a, three snow spectrums are plotted from single pixels located within the boxes in Figure $2 b$, representing areas in the image with different $r_{\mathrm{e}}$. Smaller grains are highlighted with the solid line box in an area where there was no water percolation. Larger grains and ice are highlighted in the dotted line box where there was water pooling at the capillary barrier then refreezing. Medium-sized grains are highlighted in the dashed line box where there was a preferential flow path. For the solid line spectrum in Figure $2 \mathrm{a}$, an example of the scaled band area $\left(A_{\mathrm{b}}\right)$ is shown, which corresponded to $r_{\mathrm{e}}=354 \mu \mathrm{m}$ using the lookup table.

\subsection{Laboratory experiments}

To demonstrate the novelty of the NIR-HSI method presented here, three laboratory-prepared snow samples with varying snow stratigraphy were analyzed initially and post-melt in the Subzero Research Laboratory (SRL) at Montana State University. The stratigraphy scenarios were homogeneous snow, a snow sample with an ice lens placed in the middle and a snow sample with fine grains sieved over coarse grains. In all cases, snow collected outside was sieved into a polystyrene box $(42.5 \mathrm{~cm} \times 42.5 \mathrm{~cm} \times 38.1 \mathrm{~cm})$ with a removable top and sidewall. The homogeneous snow sample was prepared by sifting snow using a $0.68 \mathrm{~mm}$ sieve. For the ice lens, snow grains were initially sifted using a $1.2 \mathrm{~mm}$ sieve, followed by fine misting water that had been chilled to $0{ }^{\circ} \mathrm{C}$ over the snow sample, such that it froze on contact creating an ice 'lens'. Once the ice lens was completely frozen, the top layer of snow grains was sifted on top of the ice lens using the same $1.2 \mathrm{~mm}$ sieve. For the snow sample with fine grains placed over the top of coarse grains, the coarse grains at the bottom were sieved with a range of $1.5-5 \mathrm{~mm}$, while the fine grains on top were sifted using a $1.2 \mathrm{~mm}$ sieve.

An initial hyperspectral image of each snow sample sidewall was taken to map $r_{\mathrm{e}}$ prior to melt, under the assumption that the stratigraphy was approximately uniform throughout the snow sample. The snow samples were then placed under a metalhalide lamp that simulates the complete solar spectrum for $\sim 6 \mathrm{~h}$, while the room temperature was held constant at $-5 \pm 1{ }^{\circ} \mathrm{C}$. The homogeneous snow sample was subjected to a simulated solar irradiance of $800 \mathrm{~W} \mathrm{~m}^{-2}$, while the ice lens and fine over coarse grain samples were subjected to a simulated solar irradiance of $650 \mathrm{~W} \mathrm{~m}^{-2}$. Once the solar lamp was turned off, the snow samples were moved to a separate storage chamber within the SRL and held at $-30^{\circ} \mathrm{C}$ for rapid refreezing. After $24 \mathrm{~h}$ at $-30{ }^{\circ} \mathrm{C}$, the sidewall of the polystyrene box was removed to reveal the snow stratigraphy, like viewing a snow pit wall in the field. Each snow sample sidewall was serial sectioned using a snow saw at intervals of $3-5 \mathrm{~cm}$, with hyperspectral images taken of the sidewall between the removal of each section. Each image was processed using the methodology outlined above in Section 2.2 to map $r_{\mathrm{e}}$ across each image. To illustrate this method, we present one imaged cross-section for each snow sample, initially and post-melt.

\subsection{Method assessment}

\subsubsection{Instrument comparison}

To assess the validity of the NIR-HSI method, the $r_{\mathrm{e}}$ retrieval from the hyperspectral imager was compared to retrieval methods using a field spectrometer and micro-CT. A homogeneous snow sample ( $1 \mathrm{~mm}$ sieved snow grains) was prepared using the method described in Section 2.3 and the snow sample sidewall was measured with each instrument for comparison. The snow sample sidewall was first imaged with the hyperspectral imager, resulting in 54570 spatial pixels, which was inverted to $r_{\mathrm{e}}$ using the method described in Section 2.2.

Next, using the same halogen light source and white reference panel, sidewall reflectance was measured using an Analytical Spectral Devices (ASD) FieldSpec 4 High Resolution field spectrometer, which has a spectral range of 350-2500 nm, and spectral bandwidth of $1.1 \mathrm{~nm}$ in the NIR. To reduce shadowing, the halogen light source was positioned to the side of the spectrometer ( $15^{\circ}$ off nadir). The field of view was reduced using an $8^{\circ}$ fore optic, which was held perpendicular to the sidewall at a distance of $\sim 4 \mathrm{~cm}$ from the snow surface, resulting in an approximate spot size of $5.6 \mathrm{~mm}$. Following a white reference measurement, 30 discrete measurements of snow reflectance were made in a space covering a grid pattern. Each spectrum was then inverted for $r_{\mathrm{e}}$ using the method described in Section 2.2 but using a separate lookup table for an illumination angle of $15^{\circ}$.

Last, five snow sub-samples were cut from the snow sample sidewall for measurement using a SkyScan 1173 micro-CT housed in a $-10^{\circ} \mathrm{C}$ cold room in the SRL. Samples were measured in a 33 $\mathrm{mm}$ diameter $\times 67 \mathrm{~mm}$ long cylindrical tube that allowed for a voxel size of $19.4 \mu \mathrm{m}$. Measurements were taken using a $42 \mathrm{kV}$, $190 \mathrm{~mA}$ X-ray beam, $100 \mathrm{~ms}$ exposure time, and each sample was rotated $180^{\circ}$ at $0.7^{\circ}$ increments. The scanning time of each sample was $\sim 25 \mathrm{~min}$ and all five samples were measured within $3 \mathrm{~h}$ of the hyperspectral imager and field spectrometer measurements. Additionally, a $3 \times 3 \times 3$ median filter was applied to the reconstructed images, thresholding of the grey-scale images into ice and air phases was performed by visual inspection, and a despeckling filter was used to remove both white and black speckles $<25$ voxels. The volume and surface area of each snow sample was measured in 3D based on the marching cubes method (Lorensen 
and Cline, 1987). The total surface area calculated from the proprietary Bruker micro-CT analysis software (CTAn) includes 'false' surface area from the volume of interest intersecting snow grains, which is believed to add a positive bias to the measured SSA. Conversely, removing the intersection surface area from the total surface area results in a negative bias to the measured SSA, whereas the actual SSA resides somewhere in the middle. Therefore, we report the average SSA value measured by including intersection surface area and removing intersection surface area to calculate $r_{\mathrm{e}}$ using Eqn (1).

To quantitatively compare $r_{\mathrm{e}}$ retrievals between each instrument, the mean, std dev. and distributions of $r_{\mathrm{e}}$ were compared and visualized. Furthermore, each measurement from the micro-CT and field spectrometer was overlaid onto the $r_{\mathrm{e}}$ map produced by the hyperspectral imager to visualize the spatial variability between all retrievals. These results are shown in Section 3.2.

\subsubsection{Sensitivity to illumination angle}

Because it is an important control on NIR reflectance, the NIR-HSI method relies on the illumination angle to be correctly accounted for when generating $r_{\mathrm{e}}$ lookup tables. At higher incidence angles, a photon will, on average, undergo its first scattering event closer to the surface which increases the probability of scattering in an upward direction, exiting the snowpack without being absorbed (Warren, 1982). Therefore, for the same $r_{\mathrm{e}}$, reflectance increases with illumination angle, resulting in smaller $A_{\mathrm{b}}$ values. For example, Figure 3 shows the directional-hemispherical reflectance at $0^{\circ}, 30^{\circ}$ and $45^{\circ}$ illumination angles for an $r_{\mathrm{e}}$ of $100 \mu \mathrm{m}$ modeled using SNICAR. In assessing the sensitivity of the retrieval of $r_{\mathrm{e}}$ to illumination angle, we note that although for modeling purposes we assume the halogen lights are at $0^{\circ}$, there are slight variations from this in our experiments. This is due to two factors, (1) the two halogen lights can be independently rotated, and (2) the lights must be placed slightly below the scanning stage to not obstruct the field of view of the imager.

The sensitivity of the $r_{\mathrm{e}}$ retrieval to variation in illumination angle was assessed by comparing consecutive images of the same homogeneous snow sample sidewall, prepared following the procedure in Section 2.2. While the hyperspectral imager remained stationary and perpendicular to the snow sample sidewall, the halogen lights were moved from $0^{\circ}$ to $45^{\circ}$ illumination angles at increments of $5^{\circ}$. To demonstrate the error that can result from not specifying the correct illumination angle, each image was processed using the lookup table for a $0^{\circ}$ illumination angle and compared to additional lookup tables generated with SNICAR for the corresponding illumination angles.

\subsubsection{Repeatability}

Multiple images of a homogeneous snow sample were also used to assess sensitivity to sensor noise and investigate the precision of the $r_{\mathrm{e}}$ retrieval. To accomplish this, the snow sample sidewall was imaged five consecutive times over a 3 min time period without any changes made to the experimental setup. To compare the consistency in $r_{\mathrm{e}}$ retrievals, we compare the range of values from each image using a box plot analysis and assess spatial differences with pixel by pixel std dev. However, since it was not possible to exactly align pixels between images, std dev. were calculated over $3.5 \mathrm{~cm}^{2}$ subregions of the image. This represents the average std dev. across $\sim 20 \times 20$ pixels in the original images.

\section{Results and discussion}

\subsection{Laboratory experiment}

In Figures $4 \mathrm{a}-\mathrm{f}, r_{\mathrm{e}}$ is shown for the three laboratory-prepared snow samples initially and post-melt. Although the lookup table

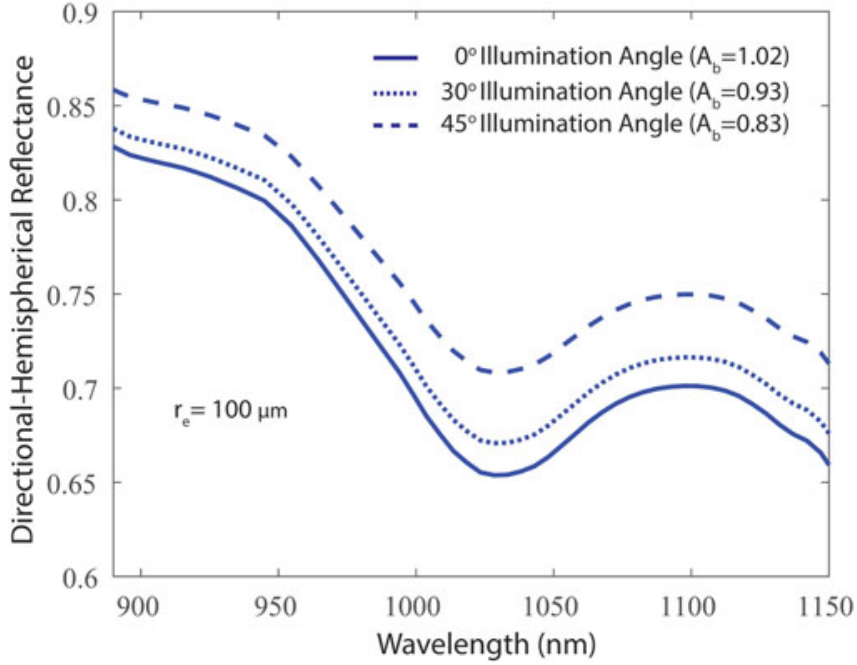

Fig. 3. Directional-hemispherical reflectance at $0^{\circ}, 30^{\circ}$ and $45^{\circ}$ illumination angles for effective grain radius $\left(r_{\mathrm{e}}\right)$ of $100 \mu \mathrm{m}$ modeled using SNICAR. Scaled band area $\left(A_{\mathrm{b}}\right)$ of the ice absorption feature centered at $1030 \mathrm{~nm}$ decreases with increasing illumination angle.

for these measurements is populated for grains with $r_{\mathrm{e}}$ ranging from 30 to $1500 \mu \mathrm{m}$, to increase contrast and improve visualization, the color bar used in Figure 4 ranges from only 30 to $1000 \mu \mathrm{m}$. Grains outside the range of the lookup table are white $\left(r_{\mathrm{e}} \geqslant 1500 \mu \mathrm{m}\right)$ and classified as ice. In Figures $4 \mathrm{~g}-\mathrm{i}$, the initial and post-melt $r_{\mathrm{e}}$ distributions are plotted to show the shift in $r_{\mathrm{e}}$ due to grain coarsening during wet snow metamorphism (Raymond and Tusima, 1979). Interestingly, the $r_{\mathrm{e}}$ maps reveal patterns in the stratigraphy profile, seen best in initial images Figures $4 \mathrm{a}-\mathrm{c}$, which are due to the laboratory sifting process. This is the first time, of which we are aware, that this pattern has been captured and shows that although still relatively homogeneous at the sample scale, the sifting process itself does introduce spatial variability.

Prior to melt, the homogeneous snow sample mean $r_{\mathrm{e}}$ was 149 $\mu \mathrm{m}$ (std dev. $(\sigma)=17 \mu \mathrm{m})$, whereas the post-melt mean $r_{\mathrm{e}}$ was 191 $\mu \mathrm{m}(\sigma=53 \mu \mathrm{m})$ (Figs $4 \mathrm{a}, \mathrm{d})$. The sample was prepared in a way that classical grain size would be homogeneous throughout the sample, and the homogeneity was also present in the mapped $r_{\mathrm{e}}$, with the corresponding distribution being relatively narrow and unimodal (Fig. 4g). Post-melt, the $r_{\mathrm{e}}$ increased and the distribution widened due to grain coarsening and wet snow metamorphism. This example demonstrates that this method can be used to quantify and map the magnitude of grain coarsening postmelt and visualize preferential flow channels in refrozen snow (Fig. 4g).

Initial mean $r_{\mathrm{e}}$ for the snow sample containing an ice lens was $211 \mu \mathrm{m}(\sigma=41 \mu \mathrm{m})$, and the post-melt mean $r_{\mathrm{e}}$ was $395 \mu \mathrm{m}(\sigma=$ $148 \mu \mathrm{m}$ ) (Figs $4 \mathrm{~b}, \mathrm{e})$. Like the homogeneous sample, the corresponding distribution (Fig. $4 \mathrm{~h}$ ) is unimodal, but with a broader $r_{\mathrm{e}}$ range for both the initial and post-melt $r_{\mathrm{e}}$ maps. In the initial image, it is evident that the shift in the distribution relative to the homogeneous sample is primarily due to the larger grains associated with the ice lens. In the post-melt image, $r_{\mathrm{e}}$ has generally increased across the full image, and the $r_{\mathrm{e}}$ map shows evidence of preferential flow channels and pooling water at the ice lens interface.

In the fine over coarse grain snow sample, the initial mean $r_{\mathrm{e}}$ was $292 \mu \mathrm{m}(\sigma=110 \mu \mathrm{m})$, while the post-melt mean $r_{\mathrm{e}}$ was 452 $\mu \mathrm{m}(\sigma=192 \mu \mathrm{m})$ (Figs $4 \mathrm{c}, \mathrm{f})$. The layer of fine grains over coarse grains is clearly visualized in the initial and post-melt $r_{\mathrm{e}}$ maps, and corresponding distributions (Fig. 4i), which were bimodal. In the post-melt image, evidence of water pooling at the interface 

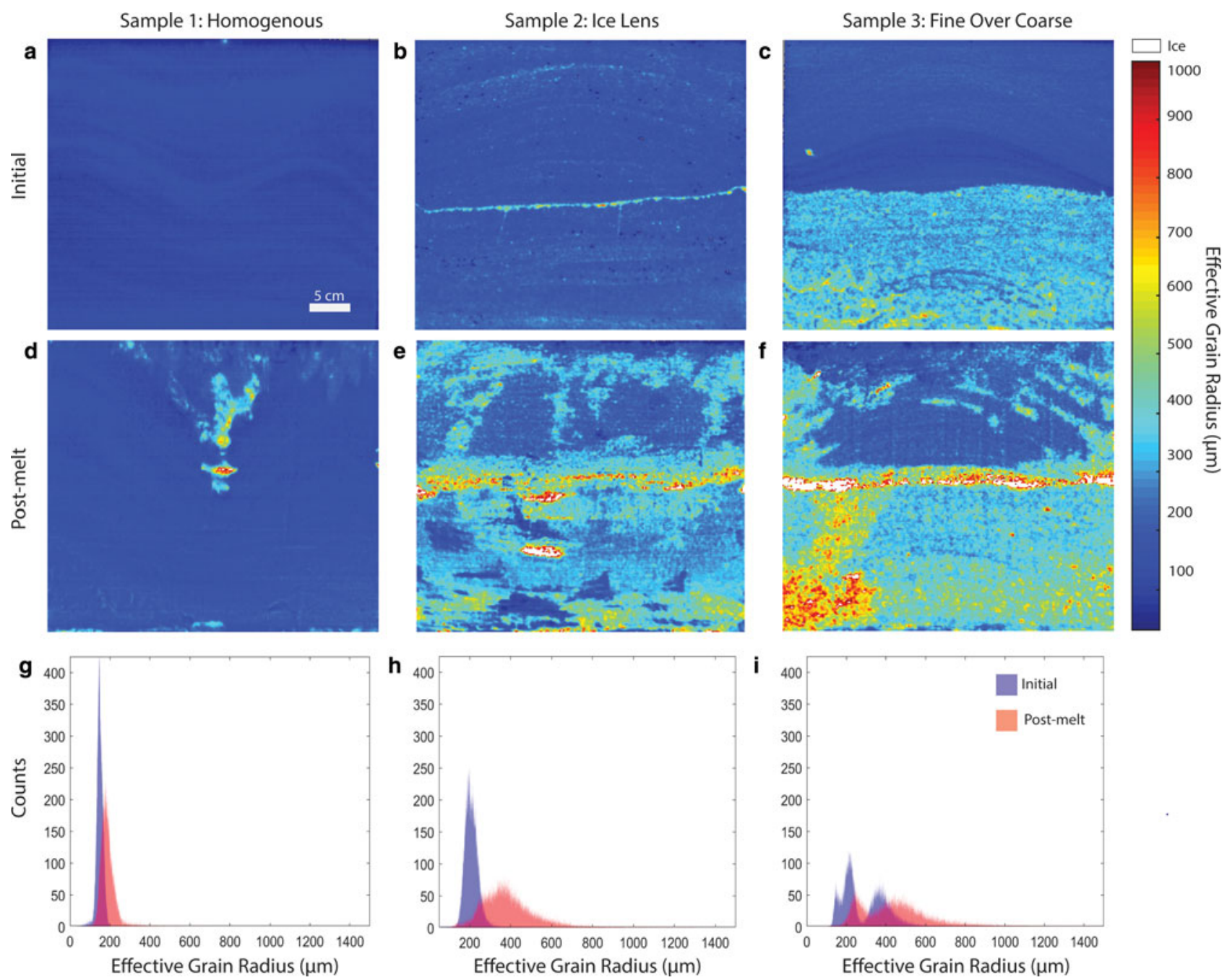

Fig. 4. (a-c) Effective grain radius $\left(r_{\mathrm{e}}\right)$ map for three laboratory-prepared snow samples; (a) homogeneous, (b) ice lens, (c) fine grains over coarse grains. (d-f) Post-melt $r_{\mathrm{e}}$ map for the three initial snow samples shown in (a-c). (g-i) Distribution of $r_{\mathrm{e}}$ for each snow sample initially, and post-melt.

between fine and coarse $r_{\mathrm{e}}$, referred to as a capillary barrier (Waldner and others, 2004), can be seen by the formation of an ice layer surrounded by larger grains. Capillary barriers in a snowpack are generally due to infiltrating water in finer grains having a very high suction, which prevents water from entering the lower (coarse grain) layer, causing local accumulation of water at the interface (ponding) and horizontal diversion of water (Yamaguchi and others, 2010; Avanzi and others, 2016).

As mentioned in Section 2.3, it should be noted that in all the hyperspectral images presented here, the snow samples had been refrozen at $-30^{\circ} \mathrm{C}$, such that there was no liquid water present (i.e. dry snow) during the data collection. This is important to reiterate because the presence of liquid water in snow does affect the ice absorption feature centered at $1030 \mathrm{~nm}$ due to the two absorption features associated with liquid water at 950 and $1150 \mathrm{~nm}$ (Nolin and Dozier, 2000). Furthermore, Green and Dozier (1996) demonstrated that high amounts of liquid water ( $25 \%$ by volume) shifted the ice absorption feature to shorter wavelengths and reduced the depth. However, Nolin and Dozier (2000) reported that for low amounts of liquid water ( $3-5 \%$ by volume), there were no effects on the accuracy of the $r_{\mathrm{e}}$ retrievals using the scaled band area (N-D) method.

\subsection{Instrument comparison}

The retrieved $r_{\mathrm{e}}$ from each instrument is shown in a box plot analysis in Figure 5a. The mean $r_{\mathrm{e}}$, std $\operatorname{dev}$. $(\sigma)$, and number of samples $(n)$ for the hyperspectral imager, field spectrometer and micro-CT, respectively, were: $r_{\mathrm{e}}=151 \mu \mathrm{m},(\sigma)=20 \mu \mathrm{m},(n)=54$ $570 ; r_{\mathrm{e}}=151 \mu \mathrm{m}, \sigma=7 \mu \mathrm{m}, n=30 ; r_{\mathrm{e}}=192 \pm 2 \mu \mathrm{m}, \sigma=12 \mu \mathrm{m}$ and $n=5$. The mean $r_{\mathrm{e}}$ value from the field spectrometer matched that of the hyperspectral imager, although the median value was $4 \%$ higher. Additionally, the full range of spectrometer $r_{\mathrm{e}}$ values generally aligned with the interquartile range of the hyperspectral imager, but the full range was not represented in the distribution. The retrieved $r_{\mathrm{e}}$ from the micro-CT fell within the upper range of $r_{\mathrm{e}}$ values from the hyperspectral imager, but the mean $r_{\mathrm{e}}$ was $23.9 \%$ higher.

The map of $r_{\mathrm{e}}$ retrievals from all three instruments is shown in Figure $5 \mathrm{~b}$. Although this snow sample was prepared to be homogeneous, the $r_{\mathrm{e}}$ map revealed slightly larger grains at the top and bottom of the snow sample. This spatial variability was not seen in field spectrometer retrievals; however, it is important to note that the spot size $(\sim 5.6 \mathrm{~mm})$ only spans a few pixels $(\sim 3 \mathrm{~mm})$ and the circles representing these measurements were only enlarged for better visualization in the figure. The micro-CT $r_{\mathrm{e}}$ measurements, which are shown approximately to scale, were higher relative to the other methods. The general trend, however, was captured across the vertical profile, as illustrated in Figure 5b.

It was expected that the $r_{\mathrm{e}}$ retrievals between the hyperspectral imager and field spectrometer would be similar given that the N-D method was applied to both instruments. This close match in mean $r_{\mathrm{e}}$ values demonstrates that the instrument specifications of the hyperspectral imager are sufficient to retrieve an accurate $r_{\mathrm{e}}$. 

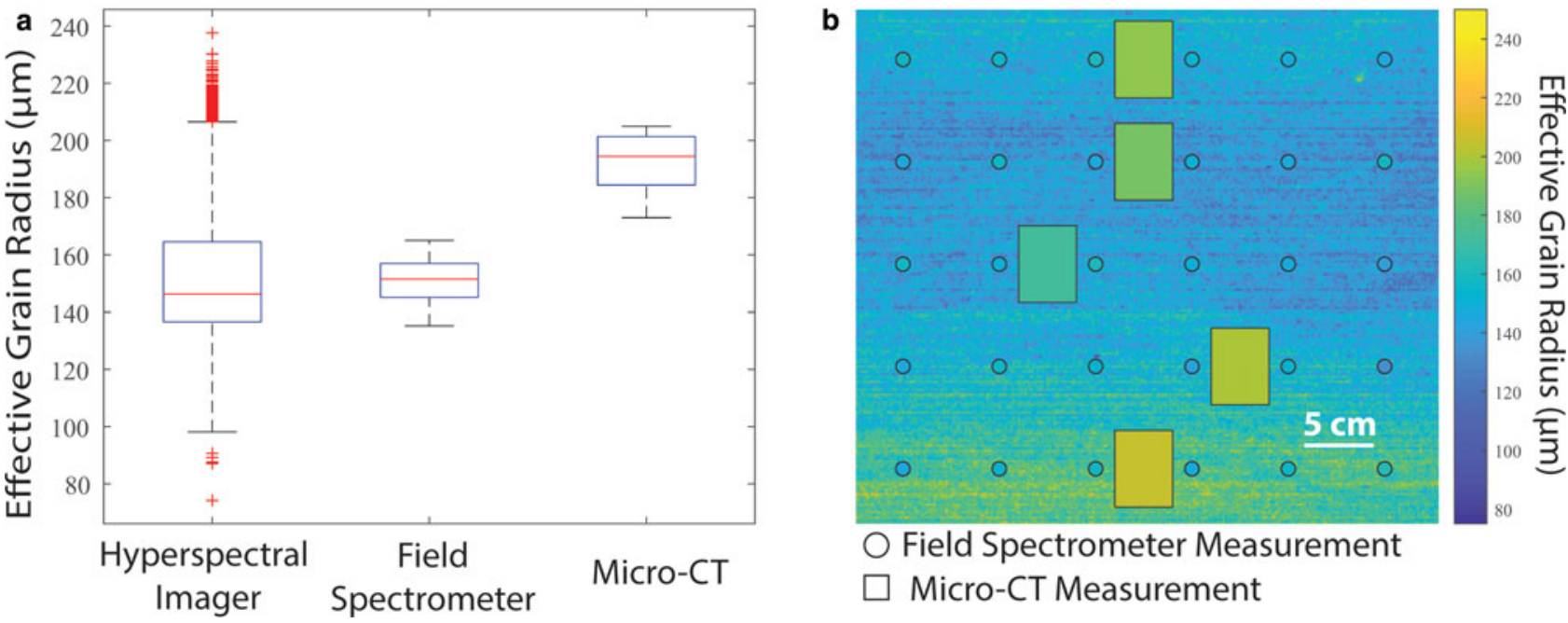

O Field Spectrometer Measurement

Micro-CT Measurement

Fig. 5. Effective grain radius $\left(r_{\mathrm{e}}\right)$ retrieval comparison between the hyperspectral imager, field spectrometer and micro-CT using a homogeneous snow sample. (a) Box plot showing the median, range and distribution of $r_{\mathrm{e}}$ from each instrument. (b) Map of $r_{\mathrm{e}}$ retrieved from the hyperspectral imager overlaid with field spectrometer and micro-CT measurements. The circles representing the field spectrometer have been enlarged for better visualization.

Provided that the field spectrometer has a significantly higher spectral resolution, broader range and better SNR than the hyperspectral imager, this is an encouraging result. The spatial variability in $r_{\mathrm{e}}$ values between the two instruments (Fig. $5 \mathrm{~b}$ ) is primarily attributed to the comparison design (i.e. fewer number of spectrometer measurements and difference in field of view), but also demonstrates the value of using a mapping technique for a more complete characterization of the $r_{\mathrm{e}}$ range and spatial variability, as evidenced in Figure 5 for the hyperspectral imager.

The discrepancy in values between the hyperspectral imager and micro-CT could be attributed to multiple factors. First, there is a fundamental difference in measurement methods. The micro-CT measures the actual snow grain surface area and volume, which is converted to $r_{\mathrm{e}}$ using the relationship in Eqn (1). Whereas the hyperspectral imager and field spectrometer are optically based methods that use a radiative transfer inversion technique based on snow grains modeled as spheres. It has been demonstrated that SSA from optical methods could have uncertainties by not accounting for grain shape (Picard and others, 2009). Additionally, there are two experimental factors that could have contributed to the discrepancy in $r_{\mathrm{e}}$ values; (1) the micro-CT measures a volume that extends $33 \mathrm{~mm}$ into the snow sample sidewall, whereas the hyperspectral imager and field spectrometer measure $r_{\mathrm{e}}$ only at the snow surface, due to the shallow penetration of light in the NIR wavelengths, and (2) the five micro-CT scans spanned $\sim 3 \mathrm{~h}$ post reflectance measurements, during which some change in the grain morphology may have occurred. These results are similar to reported values in Gergely and others (2014), where $r_{\mathrm{e}}$ values retrieved from NIR reflectance measurements using the Infrasnow instrument were within $25 \%$ of micro-CT measurements. Although Nolin and Dozier (2000) report a much better agreement $(r=0.997)$ between retrieved $r_{\mathrm{e}}$ and measured $r_{\mathrm{e}}$, we cannot compare these micro-CT results to those because they do not specify which of their measured grain radii are from stereology versus hand lens.

\subsection{Sensitivity to illumination angle}

The primary goal of the illumination sensitivity experiment was to assess how deviations in the light source illumination angle from nadir $\left(0^{\circ}\right)$ could impact the NIR-HSI method $r_{\mathrm{e}}$ retrieval. As noted in Section 2.4.2, images were progressively collected across

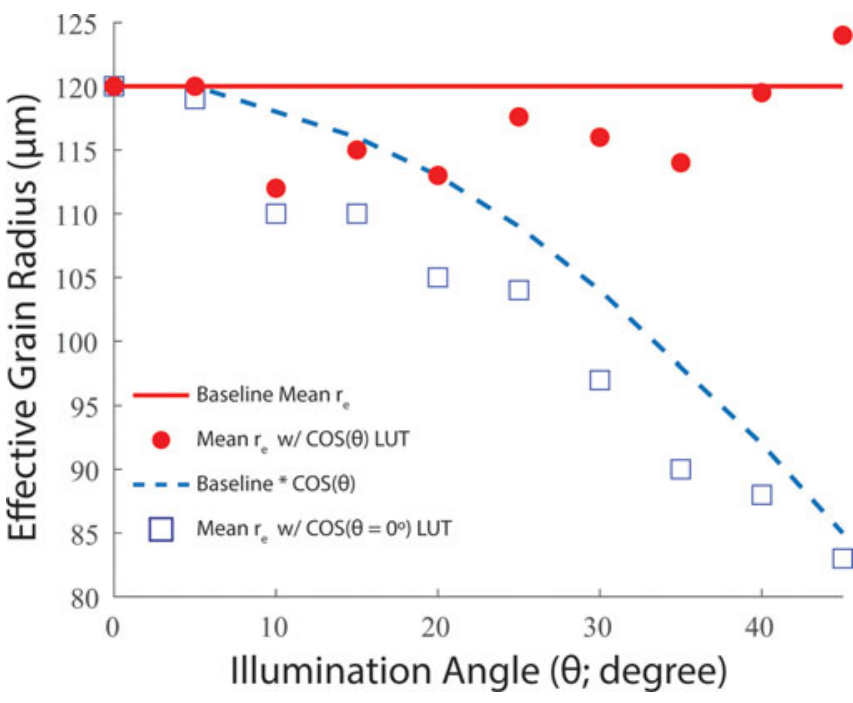

Fig. 6. Sensitivity of illumination angle to the effective grain radius $\left(r_{\mathrm{e}}\right)$ retrieval method. Retrieved mean $r_{\mathrm{e}}$ using the lookup table generated for nadir illumination $\left(\theta=0^{\circ}\right)$ is plotted as a function of illumination angle as blue squares. The decrease is relatively proportional to the baseline $r_{\mathrm{e}}$ multiplied by the cosine of the illumination angle (dashed blue line). Mean $r_{\mathrm{e}}$ retrievals using a lookup table generated with the correct illumination angle are plotted as red circles and compared to the baseline mean $r_{\mathrm{e}}$ from nadir illumination (red line).

increasing illumination angles, and $0^{\circ}$ was treated as the baseline against which all other retrievals were compared. The baseline image mean $r_{\mathrm{e}}$ was $120 \mu \mathrm{m}$ (red line in Fig. 6). When the $0^{\circ}$ lookup table was used at all other angles the retrieved $r_{\mathrm{e}}$ decreased relative to the baseline image, from $119 \mu \mathrm{m}$ at $5^{\circ}$ to $83 \mu \mathrm{m}$ at $45^{\circ}$, with the decrease being relatively proportional to the cosine of the illumination angle (Fig. 6, squares and dashed blue line). For the $r_{\mathrm{e}}$ retrievals presented in Section 3.1, we assumed the halogen light source was at a $0^{\circ} \pm 5^{\circ}$ and processed images with a $0^{\circ}$ illumination angle lookup table. The deviation of mean $r_{\mathrm{e}}$ values was $<1 \%$ between $0^{\circ}$ and $5^{\circ}$, indicating that slight deviations from $0^{\circ}$ do not significantly impact the $r_{\mathrm{e}}$ retrieval, but that larger deviations would introduce a bias toward smaller $r_{\mathrm{e}}$ (Fig. 6).

On the other hand, comparable mean $r_{\mathrm{e}}$ values to the baseline image were retrieved across all illumination angles included in the experiment when individual lookup tables were generated for each respective illumination angle tested (Fig. 6, circles). The difference 

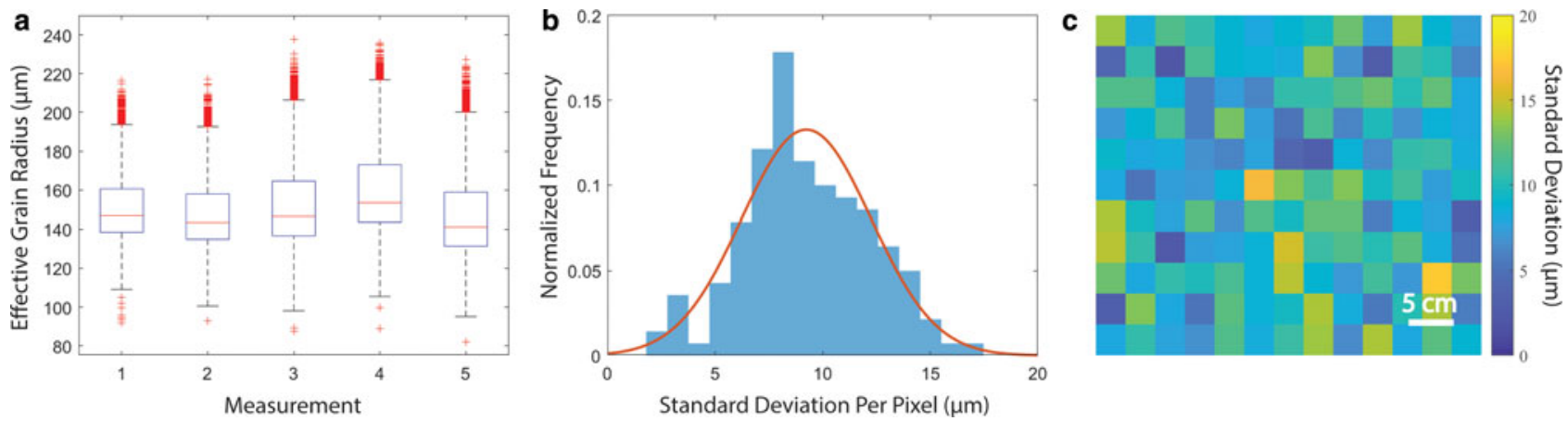

Fig. 7. Hyperspectral imager repeatability results to assess sensitivity to sensor noise. (a) Box plot analysis of five consecutively obtained images. (b) Histogram of the per pixel $\left(3.5 \mathrm{~cm}^{2}\right.$ subregion) std dev. of mean effective grain radius $\left(r_{\mathrm{e}}\right)$ from the snow sample sidewall imaged, (c) map of $r_{\mathrm{e}}$ standard deviations across the snow sample sidewall subregions.

between mean $r_{\mathrm{e}}$ at increasing illumination angles, relative to the baseline image, was $0 \mu \mathrm{m}$ at $5^{\circ}$ and then ranged from $-8 \mu \mathrm{m}$ at $10^{\circ}$ to +4 at $45^{\circ}$. Although the error between these results and the baseline data are within the repeatability range of this method, discussed further in Section 3.4, having an off-nadir illumination angle introduces angular uncertainties into the retrieval.

In the NIR wavelengths, snow reflectance is anisotropic, preferentially scattering light in the forward direction (Warren, 1982). This can impact the accuracy of retrieving $r_{\mathrm{e}}$ from measured directional reflectance (Painter and Dozier, 2004a, 2004b). When the illumination angle is nadir, however, snow reflectance is essentially Lambertian (Dumont and others, 2010). In our experimental setup, the uncertainty due to angular effects was minimized by keeping the view and illumination angles nadir. If both the view and illumination angles were off-nadir, using a radiative transfer model that computes angular intensities, like DISORT, would be recommended.

Lastly, we recognize that the lights used in these experiments introduce some level of uncertainty, even if minor, because the illumination may vary slightly between images. The method could be improved by modifying the light source to minimize slight variations in illumination angle. For example, lights could be fully diffused and/or co-aligned with the imager and evenly spaced around the lens. This would allow the lights to rotate with the imager, resulting in a better quasi-monostatic measurement.

\subsection{Repeatability}

The box plot analysis, shown in Figure 7a, shows that the general distribution of $r_{\mathrm{e}}$ values is similar across all five consecutive images used to test repeatability. The interquartile range for each image spans $\sim 20-30 \mu \mathrm{m}$, while the full range is $\sim 110-220$ $\mu \mathrm{m}$. The mean $r_{\mathrm{e}}$ ranged from 147 to $161 \mu \mathrm{m}$ across the five images and was $151 \mu \mathrm{m}$ for all images with a std dev. of $5 \mu \mathrm{m}$. Among all images, image 4 stands out as having a slight shift to higher values.

To assess the spatial variability across repeated images, the std dev. of the mean $r_{\mathrm{e}}$ was calculated in $3.5 \mathrm{~cm}^{2}$ subregions $(20 \times 20$ pixels). The distribution of std dev. is shown in Figure $7 \mathrm{~b}$ and a map of std dev. corresponding to the snow sample sidewall is shown in Figure 7c. The std dev. are randomly distributed across the image, indicating that the repeatability is most likely due to sensor noise, which can be approximated as Gaussian (Fig. 7b). The mean std dev. across subregions was $9 \mu \mathrm{m}$, which we use as the repeatability of the instrument. This repeatability is lower than the error due to sensor noise from AVIRIS bands $( \pm 10-50$ $\mu \mathrm{m})$ reported by Nolin and Dozier (2000), which can be attributed to the Resonon imager having more spectral bands across the ice absorption feature and a higher SNR.

\section{Conclusion and future applications}

We presented a new high spatial resolution NIR-HSI method to map $r_{\mathrm{e}}$ from spectral reflectance data measured with a Resonon Pika NIR-320 compact hyperspectral imager. To demonstrate the effectiveness of the method over a wide range of $r_{\mathrm{e}}$, the method was applied to laboratory-prepared snow samples that had undergone wet snow metamorphism induced by a simulated solar lamp. Our results demonstrate that the NIR-HSI method can be a valuable tool for (1) quantifying spatial and temporal variability in $r_{\mathrm{e}}$, (2) measuring change in grain size, (3) investigating wet snow metamorphism under refrozen conditions and (4) visualizing liquid water flow in a refrozen snowpack.

Additionally, the NIR-HSI method was compared to $r_{\mathrm{e}}$ retrievals from a field spectrometer and micro-CT. It was found that the hyperspectral imager $r_{\mathrm{e}}$ retrieval compared well to the higher spectral resolution field spectrometer but was better able to capture spatial variability due to the extensive number of data points produced from the mapping technique. Although it was found that micro-CT mean $r_{\mathrm{e}}$ values were $23.9 \%$ higher than that of the hyperspectral imager, the vertical profile followed the same spatial trend seen in the $r_{\mathrm{e}}$ map produced by the NIR-HSI method.

This robust high-resolution mapping method fills a gap in current $r_{\mathrm{e}}$ retrievals. There is increasing commercial availability of compact hyperspectral imagers, at feasible size and weight for laboratory and field applications, which will make this method more accessible. Although outside the scope of this paper, this method could be extended into the field to map $r_{\mathrm{e}}$ in an excavated snow pit or at the surface when mounted on an unmanned aerial vehicle. For field applications, careful consideration of illumination conditions would be important for accurate $r_{\mathrm{e}}$ retrievals. Mapping $r_{\mathrm{e}}$ at the snow surface would require lookup tables to be generated for the solar zenith angle at the time of measurement. In a snow pit, mapping $r_{\mathrm{e}}$ becomes more challenging because the direct and diffuse light ratio is unknown and would require some approximations to be made when generating the lookup table.

Supplementary material. The supplementary material for this article can be found at https://doi.org/10.1017/jog.2020.68

Acknowledgements. This research was supported by the NASA New Investigator Program Award 80NSSC18K0822. Additionally, Skiles was supported by the University of Utah GCSC, SWC, and Nexus. We acknowledge Resonon Inc. for supplying the hyperspectral imager used in this study. We also thank James Dillon and Ladean McKittrick for their assistance in the laboratory. We also acknowledge the use of the Subzero Research Laboratory in the Department of Civil Engineering at Montana State University. We thank Ghislain Picard and Henning Löwe for providing insightful comments that helped improve this manuscript. The presented data are available upon request from the lead author. 


\section{References}

Arnaud L and 7 others (2011) Measurement of vertical profiles of snow specific surface area with a $1 \mathrm{~cm}$ resolution using infrared reflectance: instrument description and validation. Journal of Glaciology 57(201), 17-29.

Avanzi F, Hirashima H, Yamaguchi S, Katsushima T and Michele CD (2016) Observations of capillary barriers and preferential flow in layered snow during cold laboratory experiments. The Cryosphere 10(5), 2013-2026.

Avanzi F, Petrucci G, Matzl M, Schneebeli M and De Michele C (2017) Early formation of preferential flow in a homogeneous snowpack observed by micro-CT. Water Resources Research 53(5), 3713-3729.

Bohren CF and Beschta RL (1979) Snowpack albedo and snow density. Cold Regions Science and Technology 1(1), 47-50.

Brucker L and 7 others (2011) Modeling time series of microwave brightness temperature at Dome C, Antarctica, using vertically resolved snow temperature and microstructure measurements. Journal of Glaciology 57(201), 171182.

Colbeck SC (1991) The layered character of snow covers. Reviews of Geophysics 29(1), 81-96

Dumont M (2010) High-accuracy measurements of snow bidirectional reflectance distribution function at visible and NIR wavelengths-comparison with modelling results. Atmospheric Chemistry and Physics, European Geosciences Union 10(5), 2507-2520.

Fierz C (2009) The international classification for seasonal snow on the ground. International Association of Cryospheric Sciences. IHP-VII Technical Documents in Hydrology $\mathrm{N}^{\circ} 83$, IACS Contribution $\mathrm{N}^{\circ}$ 1, Paris: UNESCO-IHP.

Flanner MG, Zender CS, Randerson JT and Rasch PJ (2007) Present-day climate forcing and response from black carbon in snow. Journal of Geophysical Research: Atmospheres 112, D11.

Flin F, Brzoska J-B, Lesaffre B, Coléou C and Pieritz RA (2004) Three-dimensional geometric measurements of snow microstructural evolution under isothermal conditions. Annals of Glaciology 38, 39-44.

Gallet JC, Domine F, Zender CS and Picard G (2009) Measurement of the specific surface area of snow using infrared reflectance in an integrating sphere at 1310 and $1550 \mathrm{~nm}$. The Cryosphere 3(2), 167-182.

Gerdel RW (1954) The transmission of water through snow. Eos. Transactions American Geophysical Union 35(3), 475-485.

Gergely M, Wolfsperger F and Schneebeli M (2014) Simulation and validation of the InfraSnow: an instrument to measure snow optically equivalent grain size. IEEE Transactions on Geoscience and Remote Sensing 52, 42364247.

Green RO and Dozier J (1996) Retrieval of surface snow grain size and melt water from AVIRIS spectra. In Proceedings of the 6th Annual JPL Airborne Earth Science Workshop, JPL Pub, Vol. 1, pp. 127-134.

Grenfell TC and Warren SG (1999) Representation of a nonspherical ice particle by a collection of independent spheres for scattering and absorption of radiation. Journal of Geophysical Research: Atmospheres 104(D24), 3169731709.

Hammonds K, Lieb-Lappen R, Baker I and Wang X (2015) Investigating the thermophysical properties of the ice-snow interface under a controlled temperature gradient: Part I: experiments \& observations. Cold Regions Science and Technology 120, 157-167.

Lorensen WE and Cline HE (1987) Marching cubes: a high resolution 3D surface construction algorithm. ACM Siggraph Computer Graphics 21(4), 163-169.

Marks D and Dozier J (1992) Climate and energy exchange at the snow surface in the Alpine Region of the Sierra Nevada: 2. Snow cover energy balance. Water Resources Research 28(11), 3043-3054.
Marsh P (1987) Grain growth in a wet arctic snow cover. Cold Regions Science and Technology 14(1), 23-31.

Matzl M and Schneebeli M (2006) Measuring specific surface area of snow by near-infrared photography. Journal of Glaciology 52(179), 558-564.

Nolin AW and Dozier J (2000) A hyperspectral method for remotely sensing the grain size of snow. Remote Sensing of Environment 74(2), 207-216.

Painter TH and 5 others (2009) Retrieval of subpixel snow covered area, grain size, and albedo from MODIS. Remote Sensing of Environment 113(4), 868-879.

Painter TH, Bryant AC and Skiles SM (2012) Radiative forcing by light absorbing impurities in snow from MODIS surface reflectance data. Geophysical Research Letters 39(17), 1-7.

Painter TH and Dozier J (2004a) The effect of anisotropic reflectance on imaging spectroscopy of snow properties. Remote Sensing of Environment 89(4), 409-422.

Painter TH and Dozier J (2004b) Measurements of the hemisphericaldirectional reflectance of snow at fine spectral and angular resolution. Journal of Geophysical Research: Atmospheres 109(D18), 1-21.

Painter TH, Molotch NP, Cassidy M, Flanner M and Steffen K (2007) Contact spectroscopy for determination of stratigraphy of snow optical grain size. Journal of Glaciology 53(180), 121-127.

Picard G, Arnaud L, Domine F and Fily M (2009) Determining snow specific surface area from near-infrared reflectance measurements: numerical study of the influence of grain shape. Cold Regions Science and Technology 56(1), $10-17$.

Pielmeier C and Schneebeli M (2003) Developments in the stratigraphy of snow. Surveys in Geophysics 24(5), 389-416.

Raymond CF and Tusima K (1979) Grain coarsening of water-saturated snow. Journal of Glaciology 22(86), 83-105.

Schneebeli M (1995) Development and stability of preferential flow paths in a layered snowpack. IAHS Publications-Series of Proceedings and Reports-International Association of Hydrological Sciences 228, 89-96.

Schneebeli M and Sokratov SA (2004) Tomography of temperature gradient metamorphism of snow and associated changes in heat conductivity. Hydrological Processes 18(18), 3655-3665.

Seidel FC, Rittger K, Skiles SM, Molotch NP and Painter TH (2016) Case study of spatial and temporal variability of snow cover, grain size, albedo and radiative forcing in the Sierra Nevada and Rocky Mountain snowpack derived from imaging spectroscopy. The Cryosphere 10(3), 1229-1244.

Stamnes K, Tsay S-C, Wiscombe W and Jayaweera K (1988) Numerically stable algorithm for discrete-ordinate-method radiative transfer in multiple scattering and emitting layered media. Applied Optics 27(12), 2502-2509.

Waldner PA, Schneebeli M, Schultze-Zimmermann U and Flühler H (2004) Effect of snow structure on water flow and solute transport. Hydrological Processes 18(7), 1271-1290.

Warren SG (1982) Optical properties of snow. Reviews of Geophysics 20(1), 67-89.

Warren SG and Brandt RE (2008) Optical constants of ice from the ultraviolet to the microwave: a revised compilation. Journal of Geophysical Research: Atmospheres 113, D14.

Wiscombe WJ and Warren SG (1980) A model for the spectral albedo of snow. I: Pure snow. Journal of the Atmospheric Sciences 37(12), 2712-2733.

Yamaguchi S, Katsushima T, Sato A and Kumakura T (2010) Water retention curve of snow with different grain sizes. Cold Regions Science and Technology 64(2), 87-93.

Zuanon N and A2 Photonic Sensors (2013) IceCube, a portable and reliable instrument for snow specific surface area measurement in the field. International Snow Science Workshop, Grenoble - Chamonix Mont-Blanc. 